\title{
Can we define migraine patients with blood high-sensitivity C-reactive protein and galectin-3 levels in the emergency department?
}

Mehtap Gürger ${ }^{1}$, Metin Atescelik ${ }^{1}$, Mustafa Yilmaz ${ }^{1}$, Mustafa Yildiz' ${ }^{1}$, Hatice Kalayci², Mehmet Ali Kobat ${ }^{3}$, Caner Fevzi Demir ${ }^{4}$

\begin{abstract}
${ }^{1}$ Department of Emergency Medicine, School of Medicine, Firat University, Elazığ, Turkey 2Department of Biochemistry, Elazığ Education and Research Hospital, Elazığ, Turkey ${ }^{3}$ Department of Cardiology, School of Medicine, Firat University, Elazığ, Turkey ${ }^{4}$ Department of Neurology, School of Medicine, Firat University, Elazığ, Turkey
\end{abstract}

Submitted: 7 September 2015

Accepted: 13 October 2015

Arch Med Sci 2018; 14, 2: 307-312

DOI: $10.5114 /$ aoms.2016.60984

Copyright @ 2016 Termedia \& Banach

\section{Abstract}

Introduction: Migraine, the most widespread cause of headache in young adults, is a frequent reason for presentation to emergency departments. The aim of this study was to determine the diagnostic value of serum galectin-3 and high-sensitivity C-reactive protein (hSCRP) levels in migraine patients.

Material and methods: Serum galectin-3 and hsCRP levels were measured using enzyme-linked immunosorbent assay in 70 migraine patients and 70 healthy control subjects.

Results: The mean values of hsCRP and galectin-3 levels were significantly higher in the migraine group than those in the control group $(1.67 \pm 0.49$, $1.12 \pm 0.38 \mathrm{mg} / \mathrm{l}, p<0.001$; and $3.76 \pm 1.45,2.05 \pm 0.29 \mathrm{ng} / \mathrm{ml}, p<0.001$, respectively). When the diagnostic values of the biomarkers were assessed in the migraine attack and control groups, the sensitivity and specificity of the test for hsCRP at the cut-off point of $1.32 \mathrm{mg} / \mathrm{l}$ were $70 \%$ and $73 \%$ (AUC $=0.805,95 \% \mathrm{Cl}: 0.74-0.88, p<0.001$ ), and for galectin-3 at the cut-off point of $2.4 \mathrm{ng} / \mathrm{ml}, 89 \%$ and $90 \%$ (AUC $=0.97,95 \% \mathrm{Cl}: 0.95-0.99, p<0.001$ ), respectively.

Conclusions: This study showed the presence of a relation between high serum levels of hsCRP and galectin-3 in migraine patients. However, well-controlled, carefully executed longitudinal studies are required to confirm these findings.

Key words: galectin-3, high-sensitivity C-reactive protein, migraine.

\section{Introduction}

Although migraine is a common reason for presentation to emergency departments (EDs), presence of this disorder is not sufficient to be diagnosed and treated [1-4]. The criteria of the International Classification of Headache Disorders-II (ICHD-II) have been found to be difficult to apply and are generally not used in the routine work-up of emergency departments, and the treatment for acute migraine attacks is usually provided with nonspecific analgesics $[1,2,5]$. At the start of migraine attacks, most migraine patients respond well to nonspecific therapy such as nonsteroidal anti-inflammatory drugs, acetaminophen plus caffeine

\author{
Corresponding author: \\ Assist. Prof. Mehtap Gürger \\ Department \\ of Emergency Medicine \\ School of Medicine \\ Firat University \\ 23100 Elazığ, Turkey \\ Phone: +90 5334616583 \\ E-mail: drmhtp@yahoo.com
}


[6]. However, migraine attacks leading to referral to EDs are usually more severe, last longer, and are relatively resistant to drugs [1]. In patients with very severe migraine attacks and not responding to nonspecific therapy, use of migraine specific drugs can be more effective $[1,2,5,7]$. Hence, the diagnosis and appropriate treatment of migraine attacks are an important issue in ED.

Galectin-3 is a lectin-binding galactoside found widely in various tissues, both intra- and extracellular, and is expressed mostly by mast cells, neutrophils, and eosinophils. This protein has been shown to be involved in biological processes such as cell adhesion, cell differentiation, and T-cell apoptosis, as well as in the activation of intracellular signal pathways in pathologies such as acute or chronic inflammation. Galectin-3, secreted by macro- and microglial cells, contributes to the migration of neuroblasts and differentiation of oligodendrocytes during brain development [8-10].

High-sensitivity C-reactive protein (hsCRP), which is an acute phase reactant produced in the liver, serves as a biomarker of systemic inflammation [11, 12]. Studies on serum hsCRP levels in migraine patients have reported controversial results. Some studies have reported higher levels of hsCRP in migraine patients than in controls [13-19], whereas others have found no significant difference in hsCRP levels between the migraine and the control groups $[11,20]$.

Although neuroinflammation has been shown to have an important role in the pathogenesis of migraine, there are only a few studies on the association of migraine with serum hsCRP level $[11,13-20]$. As far as we know, there is only one study on the association of migraine with serum galectin-3 level [21, 22]. In our study, we assessed and compared the serum levels of hsCRP, which is a biomarker of systemic inflammation, and galectin-3, which is associated with inflammation in attack- or interictal-stage migraine patients presenting to the ED and in healthy controls. The purpose of our study was to determine the diagnostic role of hsCRP and galectin-3 levels in patients presenting to the ED with an acute migraine attack.

\section{Material and methods}

Study design, inclusion and exclusion criteria

This prospectively planned case-control study was started after obtaining the approval of the local ethics committee. This study included age- and sex-matched 70 migraine patients and 70 controls. The migraine attack group consisted of 70 consecutive patients with previously diagnosed migraine who were admitted to the emergency room with headache and diagnosed as having migraine attack as a result of medical examination. The healthy control group consisted of hospital staff with no diagnosed migraine or chronic disease. All groups were evaluated by emergency physicians and neurologists. Data comprising demographic data of the patients, diagnosed diseases and drugs were recorded on a standard data record form.

The subjects were diagnosed for migraine by a clinical neurologist using a detailed questionnaire in accordance with the International Classification of Headache Disorders-II [19]. Questions used to define migraineurs included length and frequency of attack; pain location, type and intensity; associated symptoms such as nausea, vomiting, phonophobia, photophobia and other visual disturbances; and other neurological symptoms. The migraine patients were divided into two groups: those with aura and those without aura. Four or more attacks per month were classified as frequent attacks, and fewer attacks were classified as rare attacks. Neither control subjects nor migraineur subjects had any medical conditions or any acute or chronic pain disorders other than the migraineurs having migraine.

The criteria for exclusion from the study were the following: any systemic disorder (coronary artery disease, hepatic insufficiency, renal insufficiency, rheumatic diseases and malignancy), age under 18, acute infection, pregnancy, and use of non-steroidal anti-inflammatory or migraine medication in the previous week.

\section{Laboratory analysis}

A specimen of $5 \mathrm{ml}$ of venous blood was obtained from each subject in the patient and the control groups. The blood specimens were centrifuged at $4000 \mathrm{rpm}$ for $10 \mathrm{~min}$ and the separated sera were placed into small tubes and stored at $-80^{\circ} \mathrm{C}$ until the time of use. In the enzyme linked-immunosorbent assay (ELISA), the commercial kits used to determine galectin- 3 and hsCRP levels were the Human Galectin-3 ELISA kit (UscnLife, Catalogue no: SEA303Hu, Wuhan, China) and the hsCRP ELISA kit (DRG Instruments $\mathrm{GmbH}$, Catalogue no: EIA-3954, Germany), respectively. Both kits were used according to the recommendations of the manufacturers. The absorbances were read on an ELX800 ELISA reader spectrometrically at $450 \mathrm{~nm}$. The automated washer Bio-tek ELX50 (BioTek Instruments, USA) was used as the plate washer. The values of intra- and inter-assay coefficient of variation (CV) for galectin-3 were $<10 \%$ and $<12 \%$, and for hsCRP these values were $<4.2 \%$ and $<4.1 \%$, respectively.

\section{Statistical analysis}

Statistical analysis was performed using the Statistical Package for the Social Sciences (SPSS 21, 
Chicago, IL, USA). Results were given as mean \pm standard deviation (SD). The $\chi^{2}$ test was used for non-measurable parameters. Student's $t$-test and Pearson's correlation test were used for normally distributed variables in the groups. The diagnostic efficacies of galectin-3 and hsCRP for migraine were examined with receiver operating characteristics curve (ROC) analyses. $P$-values less than 0.05 were considered significant.

\section{Results}

The study included 70 migraine patients and 70 healthy controls with similar age and gender. The mean age of the migraine and control group was $36.6 \pm 9.9$ years and $34.5 \pm 8.7$ years, respectively. $71.1 \%$ of the migraine group and $72.9 \%$ of the control group were female. There was no significant difference between the two groups in terms of age and gender $(p \geq 0.05)$. Twenty-two (31.4\%) patients had frequent attacks and 10 (14.3\%) patients had attacks with aura. The patients had the migraine disease for a mean of $9.8 \pm 5.7$ years.

\section{High-sensitivity C-reactive protein and migraine}

The mean hsCRP level in migraine patients was significantly higher than that in the controls (1.67 $\pm 0.49,1.12 \pm 0.38 \mathrm{mg} /$, respectively; $p<0.001$ ). When the diagnostic efficacy of hsCRP in the migraine attack group was assessed, at a cut-off value of $1.32 \mathrm{mg} / \mathrm{l}$, the sensitivity and specificity were determined as $70 \%$ and $73 \%$, respectively (area under curve (AUC) $=0.805,95 \%$ confidence interval $(\mathrm{Cl})$ : 0.74-0.88, $p<0.001)$.

There was no statistically significant difference between the cases with frequent attacks and cases with rare attacks in terms of the hsCRP level $(1.78 \pm 0.53 \mathrm{mg} / \mathrm{ml}$ and $1.62 \pm 0.47 \mathrm{mg} / \mathrm{ml}$, respectively, $p=0.18)$. There was no significant difference between the cases with aura and cases without aura in terms of the hsCRP level $(1.85 \pm 0.29 \mathrm{mg} / \mathrm{l}$ and $1.64 \pm 0.51 \mathrm{mg} / \mathrm{l}$, respectively; $p=0.19$ ). There was no correlation between the duration of the disease and hsCRP $(r=0.189, p=0.12)$ either.

\section{Galectin-3 and migraine}

The mean galectin-3 level in the migraine patients was higher than that in the controls (3.76 $\pm 1.45,2.05 \pm 0.29 \mathrm{ng} / \mathrm{ml}$, respectively $p<0.001)$. When the diagnostic efficacy of galectin- 3 in the migraine attack group was assessed, at a cut-off value of $2.4 \mathrm{ng} / \mathrm{ml}$, the sensitivity and specificity were determined as $89 \%$ and $90 \%$, respectively (AUC $=0.97,95 \% \mathrm{Cl}: 0.95-0.99, p<0.001)$. The ROC curves of galectin- 3 and hsCRP in the migraine attack and the control groups are presented in Figure 1. Comparing their area under the
ROC curve, galectin-3 was better than hsCRP in diagnostic accuracy for migraine attack $(p<0.001)$.

There was no significant difference between the cases with aura and cases without aura in terms of the galectin-3 level (4.02 $\pm 1.05 \mathrm{ng} / \mathrm{ml}$ and $3.72 \pm 1.5 \mathrm{ng} / \mathrm{ml}$, respectively; $p=0.54$ ). Furthermore, there was no statistically significant difference between the cases with frequent attacks and cases with rare attacks in terms of the galectin-3 level (3.78 $\pm 1.61,3.75 \pm 1.38$, respectively; $p=0.94)$. There was no correlation between the duration of the disease and galectin-3 $(r=0.33$, $p=0.77$ ), either. The levels of galectin-3 and hsCRP in the patient and the control groups are presented in Figures 2 and 3.

\section{Discussion}

This study is the first to assess the diagnostic value of the biomarkers hsCRP and galectin- 3 in migraine. We observed that galectin-3 was more specific and sensitive than hsCRP in differentiating the migraine attack group from the control group. The increased response to stimuli and activation of the primary afferent nociceptors innervating the intracranial meninges contribute to the occurrence of migraine attacks [23, 24]. Among the mediators thought to activate and sensitize nociceptors are the proinflammatory cytokines released by immune cells such as tumor necrosis factor (TNF- $\alpha$ ), interleukin- $1 \beta$, and IL- 6 , in addition to histamine, serotonin, and nerve growth factor. It has been shown that these mediators increase in the internal jugular vein and serum in the first hours of the migraine attack $[23,25]$. The activation of immune cells such as macrophages and

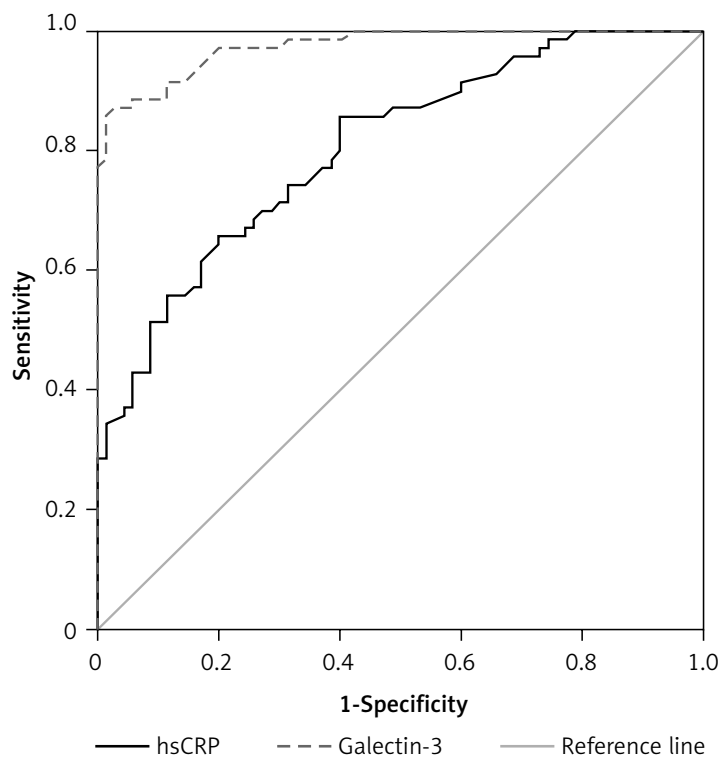

Figure 1. ROC curves of hsCRP and galectin- 3 in migraine attack group and controls 


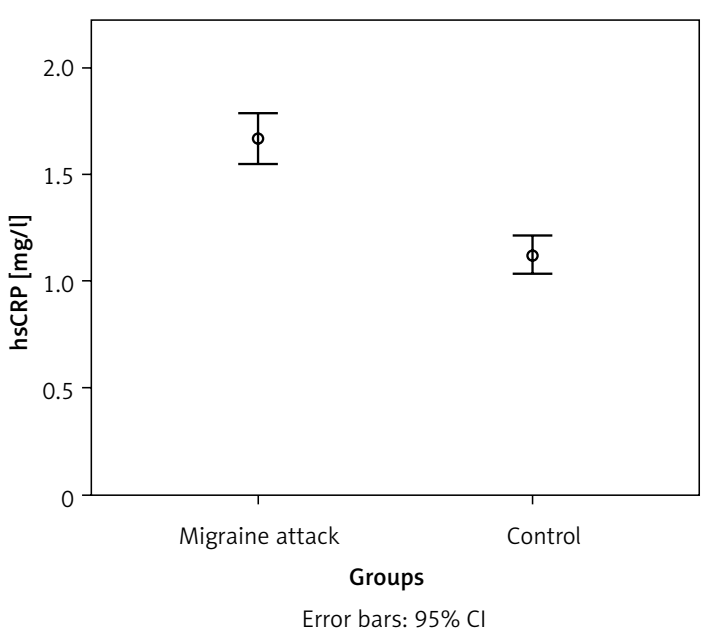

Figure 2. Serum levels of hsCRP in the migraine attack and control groups

mast cells may be the critical step in providing oversensitivity in the nociceptors [25].

Galectin-3 is a $\beta$-galactoside-binding lectin of about $30 \mathrm{kDa}$ weight [26]. It is associated with fibrosis, inflammation, and proliferation, and plays an important role in chronic inflammation [26-28]. Elevated galectin-3 levels in the blood circulation have been found to be associated with various disorders such as cancer, immunocompromised states, and cardiovascular diseases [27]. It has been reported that serum galectin-3 increases in inflammation and infections [9]. There is increased expression of galectin-3 in streptococcal pneumonia, hepatitis B infection, hepatocellular carcinoma, hepatic cirrhosis, lung injury due to radiation, and chronic pancreatitis [26].

Neuroinflammation plays an important role in the pathogenesis of migraine disease. Galectin-3 is an important pro-inflammatory mediator associated with acute and chronic inflammation. Recent studies have shown that galectins play a key role in neuroinflammation. In their study on 59 migraine patients, Yücel et al. [21] reported that galectin-3 levels in migraine patients are significantly higher than those in the controls, and that there is no significant difference in galectin-3 levels between attacks with and without aura. We also found that the galectin-3 level was higher in the migraine patients than in the controls, and that there was no statistically significant difference in the galectin-3 level between patients with frequent attacks and rare attacks, or between patients with aura and without aura. We observed no correlation between the duration of the migraine disease and galectin-3. The test for galectin-3 in patients presenting with migraine attack showed sensitivity and specificity of $89 \%$ and $90 \%$, respectively. The results indicate that the serum galectin-3 level is a good biomarker in migraine patients with attack presenting to the ED.

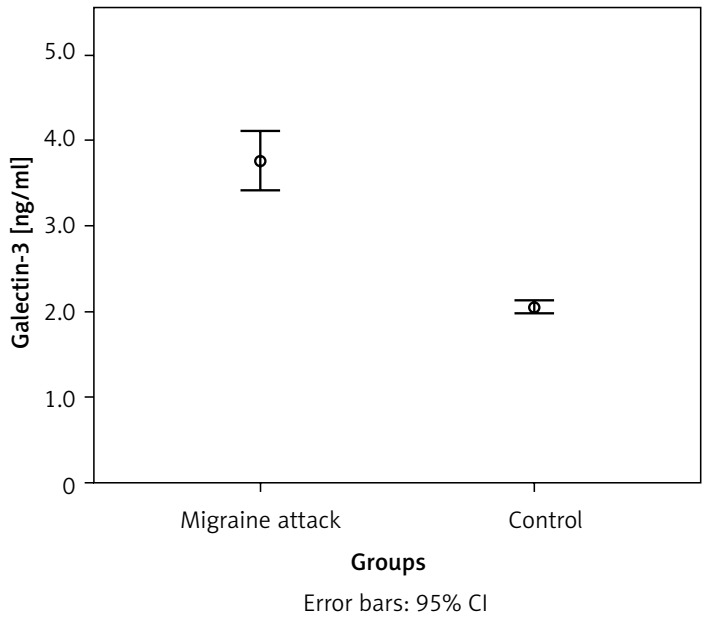

Figure 3. Serum levels of galectin-3 in the migraine attack and control groups

High-sensitivity C-reactive protein is a potential biomarker of systemic inflammation and a non-specific acute phase reactant in infection, inflammation, and various forms of tissue injury $[13-15,29]$. In their study on 125 female patients with migraine, Tietjen et al. [13] found that the hsCRP levels in migraine patients were higher than those in the controls and in patients with aura than in patients without aura, but with no significant difference. In their study on 50 young adult patients with migraine, Vanmolkot and Hoon [14] reported higher levels of hsCRP in the patient group when compared to controls, and stated that this higher value of hsCRP demonstrates the probable role of inflammation in migraine. Similarly, Avci et al. [17] and Tanik et al. [18] found that the hsCRP level in migraine patients was higher compared to the control group. Analyzing 17 different studies, Lippi et al. found that the hsCRP level in migraine patients was significantly higher compared to the control groups [19]. Salehi et al. [15] observed higher levels of $\mathrm{CRP}$ in migraine patients than in healthy controls. Scher et al. found no difference between migraine patients and healthy controls in terms of the CRP levels. Salehi et al. [15] claimed that the difference in hsCRP levels may change according to different populations studied and may be due to differences in the criteria of inclusion and exclusion. Welch et al. [16] found high levels of hsCRP in 60 migraine patients. Güldiken et al. [11] in their study on 50 migraine patients found no significant elevation of hsCRP in the patients when compared with the controls and no significant association of hsCRP with the attack frequency. In our study, we found that hsCRP was significantly higher in migraine patients than in controls. We found no significant association between the hsCRP level and attacks with and without aura and no correlation between the 
duration of the disease and hsCRP, either. Furthermore, there was no statistically significant difference between the patients with frequent attacks and patients with rare attacks in terms of the hsCRP level. In the migraine attack group, the sensitivity and specificity of hsCRP were $70 \%$ and $73 \%$, respectively.

Although migraine, the most widespread cause of headache in young adults, is one of the frequent reasons for presentation to emergency departments (ED), studies have shown that the limited information about the pathophysiology of this disorder may lead to diagnostic and therapeutic challenges. The doctors at the EDs are generally focused on excluding life-threatening causes of secondary headache and do not often use the criteria of ICHD-II. Furthermore, in the EDs, the need for prophylaxis is overlooked, and most of the patients are discharged without receiving a discharge diagnosis and prescription of recommended medications. This situation leads to the insufficient treatment of migraine patients at the EDs and recurrent emergency presentations, as well as having negative implications for the patient's life and socio-economic status [1-5, 30]. The use of a biochemical marker in addition to the characteristics and neurologic features of headache might be beneficial in the diagnosis of acute migraine at EDs, which, as a result, might lead to proper treatment and reduce the frequency of admission to EDs by providing prophylactic treatment

This study has some limitations. We found a difference between biomarker (hsCRP and galectin-3) levels between migraineurs and control subjects. Larger and prospective studies are needed to investigate their probable role as biomarkers of migraine. Another limitation is that we did not follow the migraineurs and control subjects in the days following the evaluation. Additionally, this study included only migraine patients who were compared with controls. Patients with other causes of primary or secondary headache were not included in this study. We are of the opinion that more comprehensive studies on the diagnosis of migraine with inclusion of other headache groups are required.

In conclusion, in this study, we found that hsCRP and galectin-3, two biomarkers playing an important role in inflammation, displayed higher levels in migraine patients than in the controls. However, we believe that more comprehensive studies should be performed in order to determine the suitability of both markers in the diagnosis of migraine.

\section{Conflict of interest}

The authors declare no conflict of interest.

\section{References}

1. Gelfand AA, Goadsby PJ. A neurologist's guide to acute migraine therapy in the emergency room. Neurohospitalist 2012; 2: 51-9.

2. Nijjar SS, Pink L, Gordon AS. Examination of migraine management in emergency departments. Pain Res Manage 2011; 16: 183-6.

3. Insinga RP, Ng-Mak DS, Hanson ME. Costs associated with outpatient, emergency room and inpatient care for migraine in the USA. Cephalalgia 2011; 31: 1570-5.

4. Maizels M. Headache evaluation and treatment by primary care physicians in an Emergency Department in the era of triptans. Arch Intern Med 2001; 161: 1969-73.

5. Torelli P, Campana V, Cervellin G, Manzoni GC. Management of primary headaches in adult Emergency Departments: a literature review, the Parma ED experience and a therapy flow chart proposal. Neurol Sci 2010; 31: 54553.

6. Bounes V, Edlow JA. Migraine: diagnosis and pharmacologic treatment in Emergency Department. Eur Rev Med Pharmacol Sci 2011; 15: 215-21.

7. Minen MA, Tanev K, Friedman BW. Evaluation and treatment of migraine in the Emergency Department: a review. Headache 2014; 54: 1131-45.

8. Shin T. The pleiotropic effecet of galectin-3 in neuroinflammation: a review. Acta Histochemica 2013; 115: 407-11.

9. Fermino ML, Polli CD, Toledo KA, et al. LPS-induced galectin-3 oligomerization results in enhancement of neutrophil activation. PLoS One 2011; 6: e 26004.

10. Baseras B, Gaida MM, Kahle N, et al. Galectin-3 inhibits the chemotaxis of human polymorphonuclear neutrophils in vitro. Immunobiology 2012; 217: 83-90.

11. Güldiken S, Guldiken B, Demir M, et al. Soluble CD40 and prolactin levels in migraine patients during interictal period. J Headache Pain 2011; 12: 355-60.

12. Kuo HS, Yen CJ, Chang CH, Kuo CK, Chen JH, Sorond F. Relation of C-reactive protein to stroke, cognitive disorders, and depression in the general population: systematic review and meta-analysis. Lancet Neurol 2005; 4: 371-80.

13. Tietjen GE, Herial NA, White L, Utley C, Kosmyna JM, Khuder SA. Migraine and biomarkers of endothelial activation in young women. Stroke 2009; 40: 2977-82.

14. Vanmolkot FH, de Hoon JN. Increased C-reactive protein in young adult patients with migraine. Cephalgia 2007; 27: 843-46.

15. Salehi H, Aminianfar M, Naenni AR, Saidi A, Rastgoo F. Comparison of serum CRP in migraine sufferers and normal population. Zahedan J Res Med Sci 2014; 16: 13-6.

16. Welch KMA, Brandes AW, Salerno L, Brandes JL. C-reactive protein may be increased in migraine patients who present with complex clinical features. Headache 2006; 46: 197-9.

17. Avci AY, Lakadamyali H, Arikan S, Benli US, Kilinc M. High sensitivity C-reactive protein and cerebral white matter hyperintensities on magnetic resonance imaging in migraine patients. J Headache Pain 2015; 16: 9.

18. Tanik N, Celikbilek A, Metin A, Gocmen AY, Inan LE. Retinol-binding protein- 4 and hs-CRP levels in patients with migraine. Neurol Sci 2015; 36: 1823-7.

19. Lippi G, Mattiuzzi C, Cervellin G. C-reactive protein and migraine. Facts or speculations? Clin Chem Lab Med 2014; 52: 1265-72.

20. Gudmundsson LS, Aspelund T, Scher Al, et al. C-reactive protein in migraine sufferers similar to that of nonmi- 
graineurs: The Reykjavik Study. Cephalgia 2009; 29: 1301-10.

21. Yücel Y, Tanriverdi H, Arıkanoglu A, et al. Increased fibrinogen, D-dimer and galectin-3 levels in patients with migraine. Neurol Sci 2014; 35: 545-9.

22. Headache Classification Committee of the International Headache Society (IHS). The International Classification of Headache Disorders, $3^{\text {rd }}$ edition (beta version). Cephalalgia 2013; 33: 629-808.

23. Zhang $X$, Burstein R, Levy D. Local action of the proinflammatory cytokines IL-1B and IL-6 on intracranial meningeal nociceptors. Cephalgia 2011; 32: 66-72.

24. Scheff NN, Gold MS. Sex differencesin the inflammatory mediator-induced sensitization of dural afferents. J Neurophysiol 2011; 106: 1662-8.

25. Levy D, Burstein R, Strassman AM. Mast cell in the pathophsiology of migraine headache: a hypothesis. Headache 2006; 46: 13-8.

26. Henderson NC, Sethi T. The regulation of inflammation by galectin-3. Immunol Rev 2009; 230: 160-71.

27. De Boer RA, Verweij N, van Veldhuisen DJ, et al. A genome-wide assocation study of circulating galectin-3. PLoS One 2012; 7: e47385.

28. Ge XN, Ha SG, Liu FT, Roa SP, Sriramarao P. Eosinophil-expressed galectin-3 regulates cell trafficking and migration. Front Pharmacol 2013; 4: 37.

29. Ertek S, Cicero A. Impact of physical activity on inflammation: effects on cardiovascular disease risk and other inflammatory conditions. Arch Med Sci 2012; 8: 794-804.

30. Valade D, Lucas C, Calvel L, et al. Migraine diagnosis and management in general emergency departments in France. Cephalalgia 2010; 31: 471-80. 